\title{
Effects of Liner Length and Attenuation on NASA Langley Impedance Eduction
}

\author{
M. G. Jones* and W. R. Watson ${ }^{\dagger}$ \\ NASA Langley Research Center, Hampton, VA 23681
}

\begin{abstract}
This study explores the effects of liner length and attenuation on the CHE (convected Helmholtz equation) impedance eduction method, in which the surface impedance of an acoustic liner is inferred through an iterative process based on repeated solutions to the convected Helmholtz equation. Wire mesh-over-honeycomb and perforate-over-honeycomb acoustic liners are tested in the NASA Langley Grazing Flow Impedance Tube, and the resultant data are processed using two impedance eduction methods. The first is the CHE method, and the second is a direct method (labeled the KT method) that uses the Kumaresan and Tufts algorithm to compute the impedance directly. The CHE method has been extensively used for acoustic liner evaluation, but experiences anomalous behavior under some test conditions. It is postulated that the anomalies are related to the liner length and/or attenuation. Since the KT method only employs data measured over the length of the liner, it is expected to be unaffected by liner length. A comparison of results achieved with the two impedance eduction methods is used to explore the interactive effects of liner length and attenuation on the CHE impedance eduction method.
\end{abstract}

\section{Nomenclature}

$A_{n}, K_{n}$
$[C],\{D\},\{G\}$
$c, \rho$
$d$
$f, \omega$
$F, N, R$
$i, k$
$L_{1}, L_{2}$
$L, H$
$M$
$p, P_{n}$
$Q(x), S$
$[U],[V],[\Lambda]$
$x, y, z$
$x$
$\Delta x$
$\theta, \chi, \zeta$
$\Psi$

$=$ complex mode coefficient and axial propagation constant

$=$ Hankel matrix, right-hand-side vector, and observed data set for KT algorithm

$=$ sound speed and density in air

$=$ core depth of liner

$=$ source frequency and angular frequency $(2 \pi f)$

$=$ objective function, number of lower-wall microphones, number of duct modes

$=$ unit imaginary number $(\sqrt{-1})$ and freespace wavenumber $(\omega / c)$

$=$ axial locations of liner leading and trailing edges

$=$ test section length and height

$=$ uniform flow Mach number

$=$ acoustic pressure field, normal acoustic pressure mode

$=R^{\text {th }}$ degree polynomial, pole of $Q(x)$

$=$ matrices of left eigenvalues, right eigenvalues, and singular values

$=$ axial, vertical, and spanwise coordinates

$=$ axial location of $I^{\text {th }}$ microphone

$=$ increment spacing between $N$ evenly spaced points

$=$ normalized acoustic resistance, reactance, and impedance

$=$ phase angle of pole

\footnotetext{
*Senior Research Scientist, Research Directorate, Structural Acoustics Branch, AIAA Associate Fellow.

†Senior Research Scientist, Research Directorate, Computational AeroSciences Branch, AIAA Associate Fellow.
} 
Superscripts

$T,-1, *$

Subscripts

num, meas, $\|\quad\| \quad=$ numerical value, measured value, complex absolute value

$=$ matrix transpose, generalized matrix inverse, complex conjugate

\section{Introduction}

Over the last three decades, the NASA Langley Research Center (LaRC) Liner Physics Team has investigated a number of methods for determining the acoustic impedance of duct liners, ${ }^{1-3}$ with the one based on the convected Helmholtz equation ${ }^{2}$ (CHE) being the most widely used. The CHE impedance eduction method assumes a uniform mean flow, and combines experimental measurements and computations to educe the test liner surface impedance. It is amenable to the evaluation of variable-impedance (e.g., axially or circumferentially segmented) liners, ${ }^{4}$ but has typically been used to evaluate uniform-impedance liners.

The CHE method has been extensively used with data acquired in the NASA Langley Grazing Flow Impedance Tube (GFIT), an acoustic waveguide built specifically for the purpose of studying acoustic liners. It is amenable to the use of higher-order mode sources, ${ }^{5}$ but has generally been used with a plane-wave source such as is present in the GFIT over the majority of the frequency range of interest. Although a number of simpler impedance eduction methods have been explored by the Liner Physics Team, ${ }^{6,7}$ the flexibility of the CHE impedance eduction method remains very attractive, especially for evaluation of complex liner configurations. It is therefore of interest to explore anomalies observed with this method.

One such concern is the focus of the current study. Specifically, anomalous behavior has often been observed at low frequencies. ${ }^{2,3,8,9}$ Often, this anomalous behavior takes the form of an unexpected increase in the resistance component. A number of hypotheses have been postulated ${ }^{3}$ as to the cause of this anomalous behavior. Watson et al. ${ }^{10,11}$ demonstrated that CHE impedance eduction results are improved by implementing an exit pressure boundary condition (instead of the previously-employed exit impedance boundary condition), and suggested that the details of the mean flow profile are particularly important when the attenuation is small. In 2013, Jones et al. ${ }^{12}$ also demonstrated difficulties with the CHE impedance eduction method when the attenuation is small, and suggested that coupling between the resistance and reactance components occurs near anti-resonance frequencies. Since the low frequencies used in the GFIT approach an anti-resonance (large magnitude in reactance), this suggests that at least a portion, but not all, of the increase in educed resistance at the lower frequencies is real and not an artifact of the eduction method. Finally, recent research has caused the authors to consider one additional possibility. Specifically, it is hypothesized that the eduction method may break down as the wavelength of the source frequency becomes too large relative to the length of the liner.

The purpose of the current study is to explore in some detail the effects of liner length and attenuation on the CHE impedance eduction process over the entire frequency range typically considered in the GFIT tests. Two acoustic liners are used for this study. Both liners are local-reacting, so their true impedances should be relatively independent of liner length. The first is a wire mesh-over-honeycomb liner, for which the surface impedance can be predicted from first principles. This surface impedance is nearly independent of Mach number. The second is a single-degree-of-freedom, perforate-over-honeycomb liner. This liner is chosen because it is commonly used in aircraft engine nacelles and its impedance varies with Mach number.

Each of these liners is tested in the GFIT, and the resultant data are processed using two impedance eduction methods. The first (the CHE method) is an indirect method by which the impedance is inferred through an iterative process based on repeated solutions to the convected Helmholtz equation. The second (the KT method ${ }^{7}$ ) is a direct method that uses the Kumaresan and Tufts algorithm to determine the axial propagation constants, then computes the impedance directly (without iteration) using the wall impedance boundary condition. The KT method only employs data measured over the length of the liner (and away from the leading and trailing edges), and is therefore expected to be unaffected by liner length as long as a sufficient number of microphones exist in the wall opposite the liner to adequately capture the axial propagation constants. ${ }^{13}$

Both liners are tested in the GFIT at frequencies from 400 to $2600 \mathrm{~Hz}$, a source sound pressure level 
(SPL) of $120 \mathrm{~dB}$, and centerline Mach numbers of 0.0 and 0.3 , and the two impedance eduction methods are used to determine the respective surface impedance spectra. Aluminum tape is used to cover increasingly larger portions of each liner at the trailing edge such that the active surface length is shortened, and the measurement and eduction process is repeated. Impedance spectra are educed for each liner configuration using the CHE method, and the results are compared against the impedance spectra educed with the KT method using the full length (no tape) liner to assess the effects of liner length and attenuation on the CHE impedance eduction method.

Section II provides a description of the experimental methods and the liner configurations considered in this study. Section III provides a description of the two impedance eduction methods (CHE and KT), and results are provided in Section IV. Finally, key conclusions are summarized in Section V.

\section{Experimental Method}

This section gives a description of the measurement apparatus (the NASA Langley Grazing Flow Impedance Tube - GFIT; see Fig. 1) used to conduct the liner tests. It also gives a brief description of the two test liners that are used to evaluate the effects of liner length and attenuation on the CHE impedance eduction.

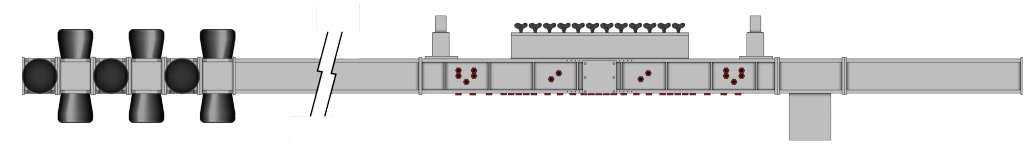

Figure 1: Sketch of NASA Langley Grazing Flow Impedance Tube (GFIT).

\section{A. Grazing Flow Impedance Tube}

The GFIT has a cross-sectional geometry of 2.0 in wide by 2.5 in high, and allows evaluation of acoustic liners with lengths from 2.0 to $24.0 \mathrm{in}$. Flow is from left to right in the figure, and is generated by the combination of a high-pressure air supply upstream and a vacuum blower downstream of the duct. The surface of the test liner forms a portion of the upper wall of the flow duct. Two source sections are available, upstream (exhaust mode, as depicted in Fig. 1) and downstream (inlet mode) of the test section. For the current study, these drivers are used to generate tones (one frequency at a time) at a source level of $120 \mathrm{~dB}$, over a frequency range of 400 to $2600 \mathrm{~Hz}$, at centerline Mach numbers of 0.0 and 0.3. Fifty-three microphones flush-mounted in the lower wall (opposite the liner) of the GFIT are used to measure the acoustic pressure field over the axial extent of $0 \leq x \leq L$ (see Fig. 2). For each data acquisition, 1000 averages on each microphone channel (1024 data points per average) are taken. A cross-spectrum signal extraction method ${ }^{14}$ is used to determine the amplitudes and phases at each of the microphone locations relative to the amplitude and phase at the reference microphone location upstream of the lined section.

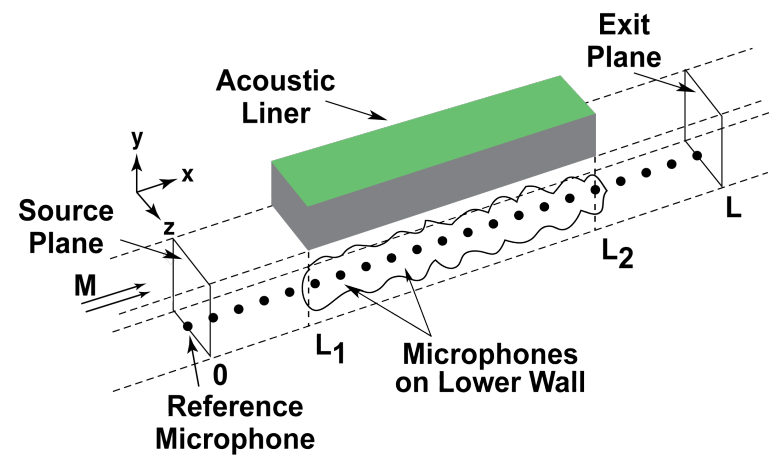

Figure 2: Sketch of Grazing Flow Impedance Tube test section. 


\section{B. Test Liners}

Two liner configurations are used in this study (Fig. 3). The first has a 2.0 in $\times 24.0$ in active surface, and consists of a wire mesh facesheet, honeycomb core, and rigid back plate. This liner is chosen because it provides an impedance spectrum that can be predicted from first principles and its resistance is expected to be independent of source SPL (i.e., linear) and Mach number. The wire mesh has a DC flow resistance of 270 MKS Rayls, which corresponds to a normalized acoustic resistance, $\theta$, of 0.65 . (Throughout this paper, all impedances are normalized by $\rho c$.) The core is 1.5 in deep, and consists of 0.375 in-diameter (distance between opposing flat surfaces) hexagonal channels. Thus, since the wire mesh facesheet can be properly treated as a lumped element, the normalized reactance, $\chi$, of this liner is given by $\chi=-\cot (k d)$, where $k=\omega / c$ is the freespace wavenumber, $d$ is the core depth, and $\omega$ is the angular frequency.

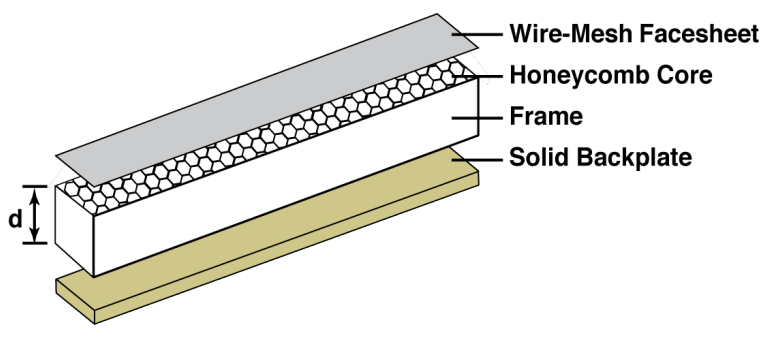

(a) Wire mesh-over-honeycomb liner.

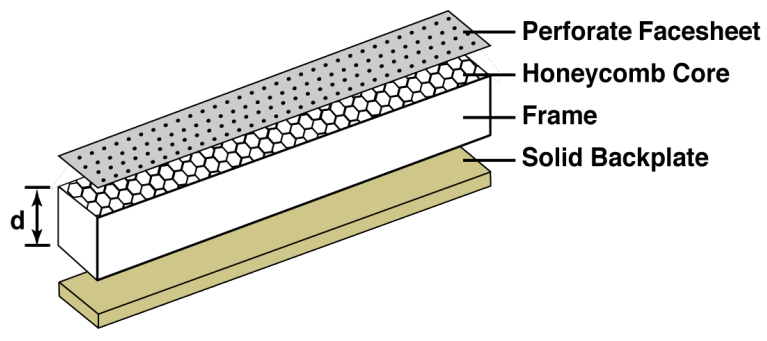

(b) Conventional, perforate-over-honeycomb liner.

Figure 3: Sketch of test liners.

The second liner is a conventional liner with a 2.0 in $\times 16.0$ in active surface, consisting of a perforate facesheet, honeycomb core, and rigid back plate. The perforate contains hole diameters of $0.039 \mathrm{in}$, a sheet thickness of $0.039 \mathrm{in}$, and a porosity of $8.7 \%$. The core is identical to that used in the wire mesh liner. This liner is weakly nonlinear, i.e., its surface impedance varies with RMS acoustic particle velocity (and hence, with source SPL). Its impedance also varies with Mach number.

It is perhaps worthwhile to note that since the conventional liner is nonlinear, the impedance will vary along the axial extent of the liner due to changes in the sound pressure level (as the liner attenuates sound). However, the eduction methods represent the entire surface as a single, average ('smeared') impedance.

\section{Impedance Eduction Methods}

Two impedance eduction methods are used in this study. The first, labeled the CHE method, is based on a finite element solution of the convected Helmholtz equation. This method uses an estimate of the liner impedance as input to a duct acoustics propagation code that predicts the SPL and phase profiles over the length of the GFIT test window (see Fig. 2). If the difference between the predicted and measured SPL and phase profiles is within an acceptable tolerance, the estimate for the impedance is taken as the unknown impedance of the liner. Otherwise, the impedance estimate is updated and the process is repeated iteratively until an impedance is found for which the difference (between measured and predicted SPL and phase profiles) is within an acceptable tolerance. The second impedance eduction method, labeled the KT method, extracts the axial propagation constant from the measured SPL and phase profiles. This allows direct computation of the impedance of the acoustic liner by substituting this measured axial propagation constant and the normal mode solution to the convected Helmholtz equation into the wall impedance boundary condition. ${ }^{7}$

\section{A. Convected Helmholtz Equation (CHE) Method}

A description of the CHE method is provided in an earlier paper by Watson, ${ }^{2}$ and is briefly summarized as follows. If the mean flow through the duct is assumed to be uniform, and only plane waves are assumed 
to be present in the spanwise direction (between the opposing hard walls), the acoustic field propagating through the flow duct (assume a time dependence of the form $e^{i \omega t}$ ) satisfies a convected Helmholtz equation on the acoustic pressure field

$$
\left(1-M^{2}\right) \frac{\partial^{2} p(x, y)}{\partial x^{2}}+\frac{\partial^{2} p(x, y)}{\partial y^{2}}-2 i k M \frac{\partial p(x, y)}{\partial x}+k^{2} p(x, y)=0
$$

where $p$ is the acoustic pressure field and $M$ is the uniform flow Mach number. The normal component of the acoustic particle velocity vanishes at the rigid lower wall, i.e.,

$$
\frac{\partial p(x, 0)}{\partial y}=0
$$

and source and exit plane boundary conditions are given by the measured acoustic pressures,

$$
p(0, y)=p(0,0) ; p(L, y)=p(L, 0)
$$

Here $p(0,0)$ and $p(L, 0)$ are the measured acoustic pressure at the lower wall microphones located at $x=0$ and $x=L$, respectively. The local-reacting wall boundary condition ${ }^{15}$ is given by

$$
-\frac{\partial p(x, H)}{\partial y}=i k\left(\frac{p(x, H)}{\zeta}\right)+2 M \frac{\partial}{\partial x}\left(\frac{p(x, H)}{\zeta}\right)+\frac{M^{2}}{i k} \frac{\partial^{2}}{\partial x^{2}}\left(\frac{p(x, H)}{\zeta}\right)
$$

where the normalized admittance, $1 / \zeta$, is taken as zero along the rigid wall portion of the upper wall and $H$ is the duct height. The CHE method of impedance eduction solves the boundary value problem defined in Eqs. (1)-(4) using a finite element method (FEM). The impedance boundary conditions are incorporated in the FEM analysis in weak form and cubic Hermite polynomials are used as the basis functions.

The unknown normalized impedance, $\zeta$, is educed by minimizing the objective function

$$
F(\theta, \chi)=\frac{1}{N} \sum_{I=1}^{N}\left\|p\left(x_{I}, 0\right)_{\text {num }}-p\left(x_{I}, 0\right)_{\text {meas }}\right\|
$$

where $N$ is the number of lower-wall microphones, $x_{I}$ is the axial location of the $I^{\text {th }}$ microphone, num denotes the numerical value obtained from the FEM, meas is the measured value, and \|\| denotes the complex absolute value.

Minimization of the objective function, $F(\theta, \chi)$, is achieved using Stewart's adaptation of the DavidonFletcher-Powell (SDFP) optimization algorithm. ${ }^{16}$ SDFP returns the normalized resistance, $\theta$, and normalized reactance, $\chi$, corresponding to the minimum point of the objective function. It has been shown ${ }^{2}$ that these extrema values correspond to the normalized impedance of the test liner $(\zeta=\theta+i \chi)$.

\section{B. Kumaresan and Tufts (KT) Method}

Application of the KT algorithm to liner impedance eduction (labeled herein as the KT method) has also been described in an earlier paper. ${ }^{7}$ Therefore, only enough detail is presented in this paper to motivate the methods and goals of the current investigation. The acoustic pressure field in the liner test section $\left(L_{1} \leq x \leq L_{2}\right)$ of Fig. 2 is expanded into a series of normal duct modes

$$
p(x, y)=\sum_{n=1}^{R} A_{n} P_{n}(y) e^{-i K_{n} x}, \quad P_{n}(y)=\cos \left(\lambda_{n} y\right), \quad \lambda_{n}^{2}=\left(k-K_{n} M\right)^{2}-K_{n}^{2}
$$

where $n$ is the mode order, $R$ is the total number of duct modes in the expansion, $A_{n}$ is the complex mode coefficient, $P_{n}(y)$ is the normal acoustic pressure mode, and $K_{n}$ is the axial propagation constant. Each term in the mode expansion [i.e., $A_{n} P_{n}(y) e^{-i K_{n} x}$ ] satisfies the convected Helmholtz equation [see Eq. (1)] and the lower wall boundary condition [Eq. (2)]. Substituting the mode solution into the wall impedance boundary condition [Eq. (4)] gives an expression for the impedance

$$
\zeta=\frac{i k}{\lambda_{n}}\left(1-K_{n} M / k\right)^{2} \cot \left(\lambda_{n} H\right)
$$


Note that if $K_{n}$ is measured using the lower wall microphone data (see Fig. 2), then Eq. (7) can be used to compute the unknown impedance, $\zeta$, of the test liner. Because each normal mode, $n$, gives the same impedance, the most accurately measured axial propagation constant, $K_{n}$, produces the most accurate impedance. This axial propagation constant typically corresponds to the least attenuated mode.

To avoid mode aliasing, a rule of thumb is that the number of modes, $R$, used in the analysis should be greater than the number of modes in the signal. Thus, some of these $R$ modes will be extraneous. ${ }^{7}$ These extraneous modes are identified via the KT algorithm, which is described briefly below.

1. Measure the acoustic pressure field at $N$ equally spaced points, $x=x_{1}, x=x_{2}, \ldots x_{N}$, on the lower wall of the flow duct.

2. From these acoustic pressure measurements, construct the prediction equations

$$
\begin{aligned}
& {[C]\{D\}=-\{G\}} \\
& {[C]=\left[\begin{array}{ccccc}
p_{2} & p_{3} & p_{4} & \cdots & p_{R+1} \\
p_{3} & p_{4} & p_{5} & \cdots & p_{R+2} \\
p_{4} & p_{5} & p_{6} & \cdots & p_{R+3} \\
p_{5} & p_{6} & p_{7} & \cdots & p_{R+4} \\
\vdots & \vdots & \vdots & \vdots & \vdots \\
p_{N-R+1} & p_{N-R+2} & p_{N-R+3} & \cdots & p_{N}
\end{array}\right],\{D\}=\left\{\begin{array}{c}
D_{2} \\
D_{3} \\
D_{4} \\
\vdots \\
D_{R+1}
\end{array}\right\},\{G\}=\left\{\begin{array}{c}
p_{1} \\
p_{2} \\
p_{3} \\
\vdots \\
p_{R}
\end{array}\right\}}
\end{aligned}
$$

where $p_{1}, p_{2}, \ldots p_{N}$, are the measured values of $p(x, 0)$ at $x=x_{1}, x=x_{2}, \ldots x=x_{N}$, respectively. These are obtained from the microphone measurements. Note that the coefficients in the Hankel matrix, $[C]$, and right-hand-side vector, $\{G\}$, are known from the observed data set.

3. Use singular value decomposition to obtain the generalized inverse of $[C]$

$$
[C]^{-1}=[V][\Lambda]^{-1}\left[U^{*}\right]^{T}
$$

Here, the superscripts $T,-1$, and $*$ denote a matrix transpose, the generalized matrix inverse, and the complex conjugate, respectively. Further, $[U],[V],[\Lambda]$ are the matrix of left eigenvalues, matrix of right eigenvalues, and matrix of singular values, respectively. The singular value decomposition will allow the normal modes and the spurious modes to be separated.

4. The generalized matrix inverse is used to obtain the vector of polynomial coefficients, $\{D\}$

$$
\{D\}=-[C]^{-1}\{G\}
$$

5. The linear prediction coefficients $D_{2}, D_{3}, \ldots D_{R+1}$ from the solution vector $\{D\}$ are used to construct the $R^{\text {th }}$ degree polynomial, $Q(X)$

$$
Q(X)=1+D_{2} X+D_{3} X^{2}+D_{4} X^{3}+\ldots D_{R+1} X^{R}
$$

It can be shown that the zeroes of $Q(X)$ are related to the axial propagation constants, $K_{n}$, in the mode expansion in Eq. (7).

6. Use a complex polynomial root finder to find the zeroes, $X$, of the polynomial defined in Eq. (12).

7. Find the measured axial wave number, $K_{n}$, from the poles, $S$, of $Q(X)$

$$
S=\frac{1}{X}=e^{i \Delta x K_{n}}=\|S\| e^{i \Psi}
$$

where $\Delta x$ is the increment spacing between the $N$ evenly spaced points and $\Psi$ is the phase angle of the pole. The axial wave numbers corresponding to poles with a magnitude inside the unit circle (i.e., $\|S\|<1$ ) are spurious while the remaining axial wave numbers (i.e., those for which $\|S\| \geq 1$ ) correspond to normal modes in the duct.

8. The acoustic impedance is computed for the most accurate normal mode (typically the least attenuated) using Eq. (7). 


\section{Results and Discussion}

\section{A. Wire mesh liner results}

Acoustic pressure data (SPL and phase profiles) were acquired with a 24"-long, wire mesh liner mounted in the GFIT. The wire mesh facesheet provides a known resistance that is virtually independent of grazing flow Mach number. As shown in Figures 4 and 5, the resistances educed at Mach 0.0 and 0.3 with the KT method are nearly constant over the majority of the frequency range at a value very near the known resistance of the wire mesh material (0.65, as corroborated via independent DC flow resistance measurements). Therefore, the $\mathrm{KT}$ results will be used as a basis for comparison with results achieved with the CHE impedance eduction method.

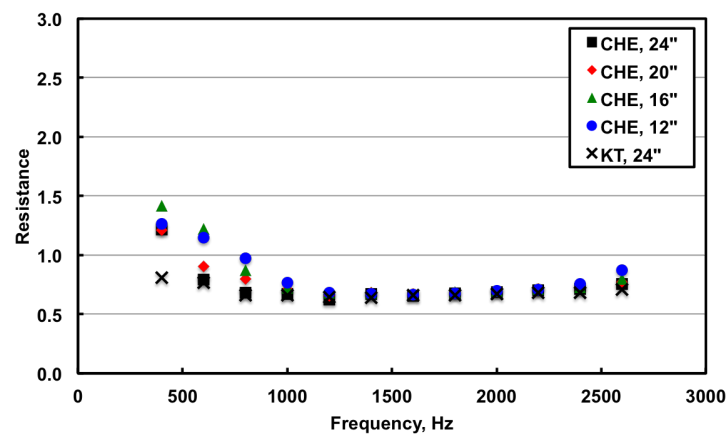

(a) Normalized resistance.

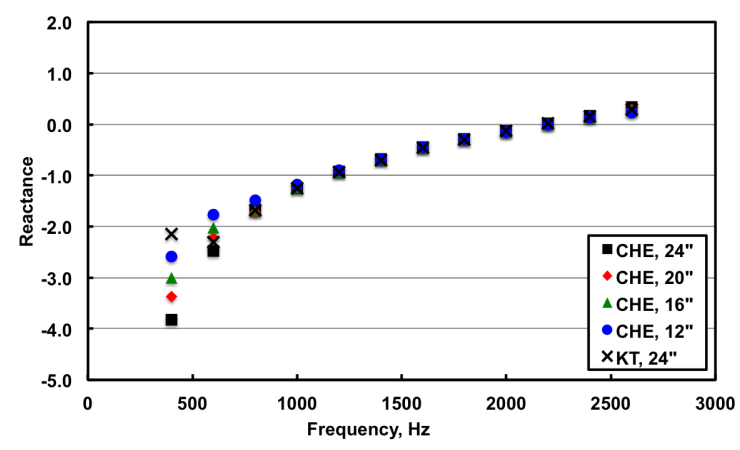

(b) Normalized reactance.

Figure 4: Comparison of impedance spectra educed with CHE and KT methods for liner lengths of 12 to 24 inches. Wire mesh liner; Mach 0.0.

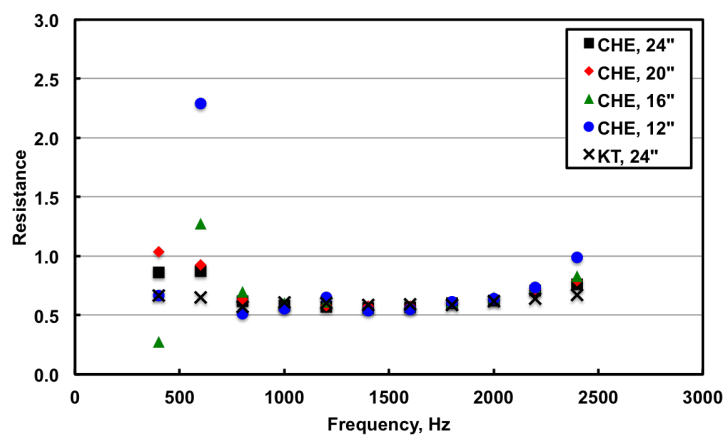

(a) Normalized resistance.

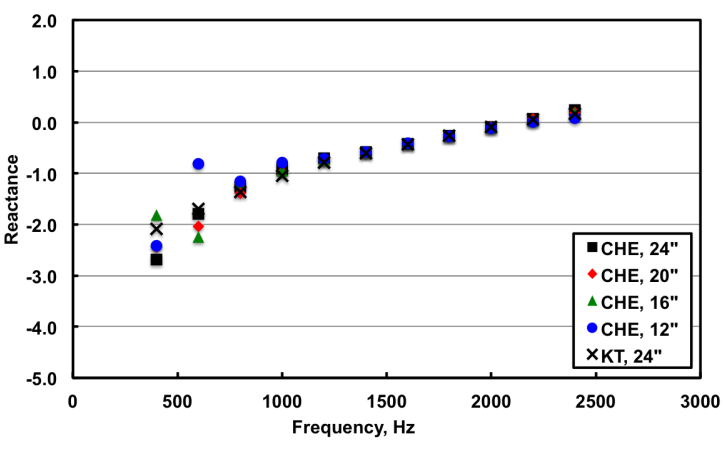

(b) Normalized reactance.

Figure 5: Comparison of impedance spectra educed with CHE and KT methods for liner lengths of 12 to 24 inches. Wire mesh liner; Mach 0.3.

Figure 4 compares impedance spectra educed with the CHE and KT methods in the absence of mean flow. The first spectrum (black squares) is educed with the CHE method for the full length liner (no tape). The remaining spectra (red diamonds, green triangles, and blue circles) represent impedances educed with the CHE method when 4,8 , or 12 inches of tape are used to cover the trailing edge of the liner, respectively. 
The black ' $\mathrm{x}$ ' symbols represent impedances educed with the KT method for the full liner. The CHE results for this liner (Fig. 4) deviate from the KT results for frequencies at or below $1000 \mathrm{~Hz}$. This holds for both components of impedance. When the Mach number is increased to 0.3 (Fig. 5), differences between the KT and CHE results remain at the low frequencies, and also become evident at the highest frequency $(2400 \mathrm{~Hz})$. The reactance is more forgiving, i.e., less susceptible to error in the eduction process.

Three reasons are considered as the cause for differences between the KT and CHE results. First, although the KT method has been chosen as a baseline against which the CHE results are compared for the purposes of this study, this does not suggest that the KT method is immune to problems of its own. Indeed, the results achieved with the KT method at $400 \mathrm{~Hz}$ deviate from the expected values. As will be shown in the following section, problems with the KT method at this frequency become much more significant when a conventional liner is used in the test. It is not clear whether this is due to the fact that the conventional liner (16" long) is shorter than the wire mesh liner (24" long), or that the conventional liner is more nonlinear. Regardless, $400 \mathrm{~Hz}$ results are not used for the more detailed comparisons to follow. As noted from previous investigations, ${ }^{11,12}$ the CHE impedance eduction method encounters difficulty when the attenuation [computed as $\operatorname{SPL}(0)-\operatorname{SPL}(L)$ ] is very low. It is also postulated that the length of the liner affects the CHE impedance eduction results. Specifically, it seems plausible that the eduction process should improve as the ratio of the test frequency wavelength to the liner length decreases. Both possible causes of error are explored in an attempt to determine their relative importance to the CHE impedance eduction method.

Figure 6 provides a comparison of attenuation levels measured with the wire mesh liner installed in the GFIT. The attenuation is generally higher for the Mach 0.0 condition, with a peak at $1600 \mathrm{~Hz}$. The peak attenuation shifts to $1800 \mathrm{~Hz}$ for the Mach 0.3 condition. (The optimum impedance is frequency and Mach number dependent.) Observe that the attenuation is quite small (approximately $5 \mathrm{~dB}$ or less) for the Mach 0.0 condition at frequencies of 400 and $600 \mathrm{~Hz}$. These frequencies correspond to those for which differences between the KT and CHE results are largest. Similar effects of limited attenuation are also observed in the Mach 0.3 data. As noted in earlier studies, ${ }^{11,12}$ there is clearly a threshold of attenuation below which the accuracy of the CHE impedance eduction method degrades.

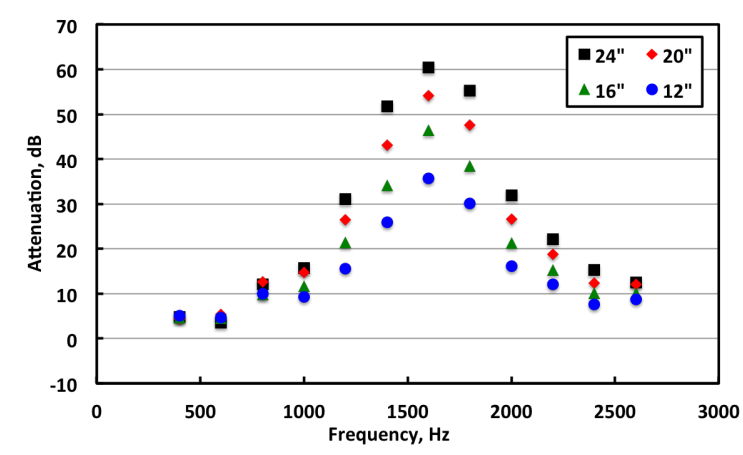

(a) Mach 0.0

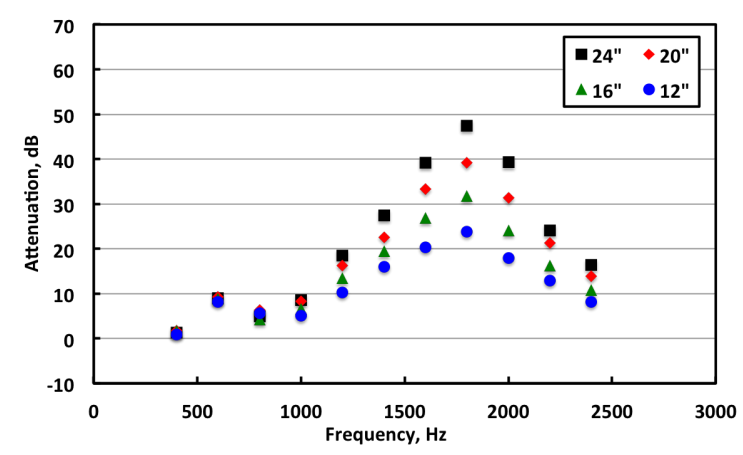

(b) Mach 0.3

Figure 6: Attenuation $[\operatorname{SPL}(0)-\operatorname{SPL}(L)]$ measured with wire mesh liner lengths of 12 to 24 inches.

Figures 7 and 8 provide comparisons of impedances educed with the CHE method for various liner lengths with the impedance educed with the KT method for the full (24") liner, for the Mach 0.0 and 0.3 conditions. The differences in resistance values, depicted as percentage errors relative to the KT results for the full length liner, are generally fairly low $(<5 \%)$. For those cases where the error is significant, it is observed to increase as the liner length is decreased. To a large extent, this observation also applies at the highest frequencies, although the amount of error is not nearly as large.

Another possibility is that an increase in the number of wavelengths that occur within the axial extent of the liner results in a decrease in the impedance eduction error. Table 1 provides a list of frequencies 
at which the wavelength is equivalent to the liner length. These 'crossover' frequencies are provided for each liner length and flow condition considered in this study. If the liner length is a dominant parameter in the impedance eduction process, one would expect the percentage error to increase as the frequency decreases below the crossover frequency, i.e., as the wavelength becomes longer than the liner length. Given the consistency of the improvement (less error) in the CHE impedance eduction method over the entire frequency range as the liner length is increased, this hypothesis appears to be valid.

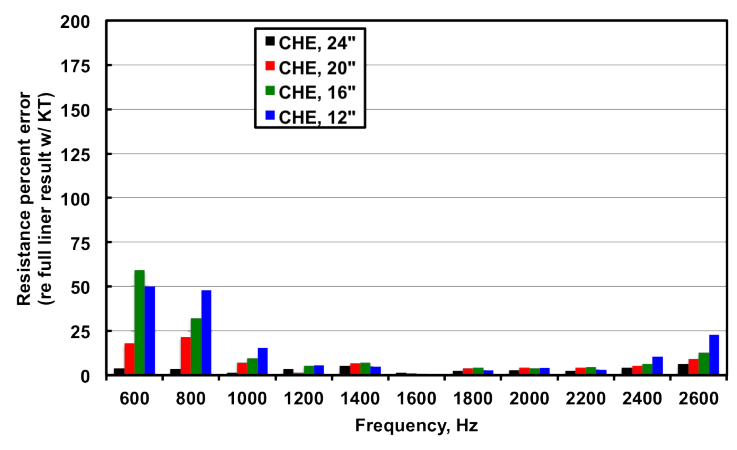

(a) Normalized resistance.

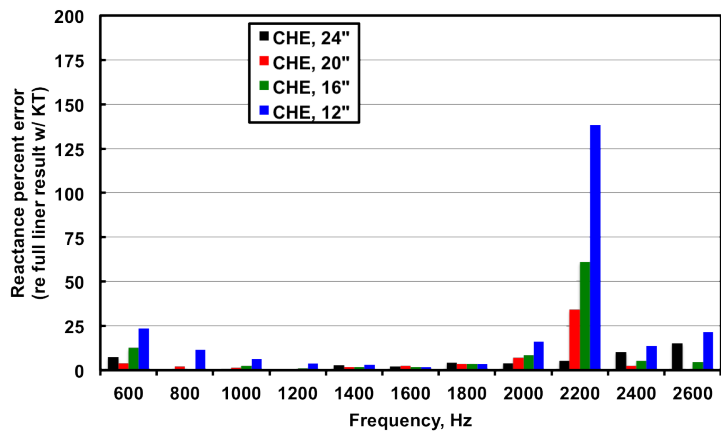

(b) Normalized reactance.

Figure 7: Comparison of CHE (for various liner lengths) and KT educed impedance spectra for full (24") wire mesh liner. Mach 0.0.

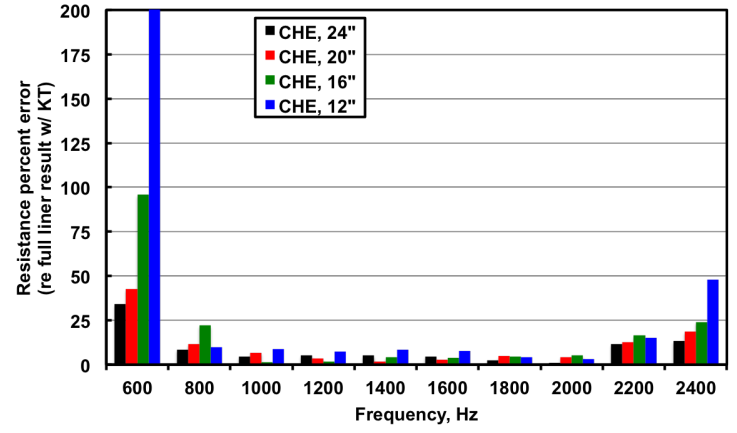

(a) Normalized resistance.

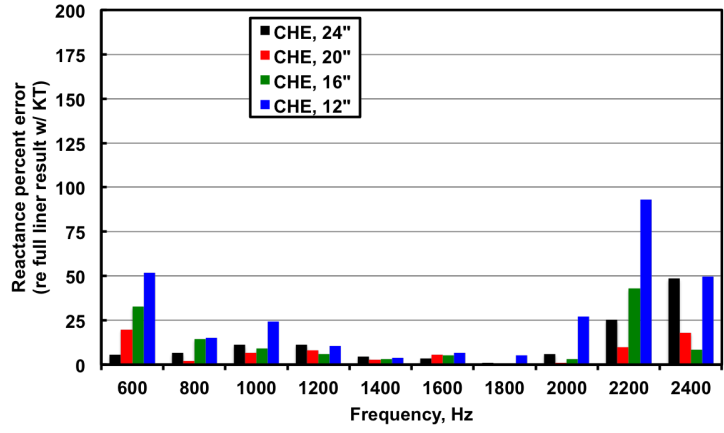

(b) Normalized reactance.

Figure 8: Comparison of CHE (for various liner lengths) and KT educed impedance spectra for full (24") wire mesh liner. Mach 0.3 .

Table 1: Crossover Frequencies

\begin{tabular}{|c|c|c|c|c|c|c|c|c|}
\hline Liner Length (in) & 10 & 12 & 14 & 16 & 18 & 20 & 22 & 24 \\
\hline$f_{c}$ at $M=0.0(\mathrm{~Hz})$ & 1356 & 1130 & 969 & 848 & 753 & 678 & 616 & 565 \\
\hline$f_{c}$ at $M=0.3(\mathrm{~Hz})$ & 1002 & 835 & 715 & 626 & 556 & 501 & 455 & 417 \\
\hline
\end{tabular}


It is also important to recall that the portions of the liner covered with aluminum tape are assumed to be rigid. Hence, even a small compliance of the aluminum tape will have a deleterious effect on the impedance eduction process with the CHE method. Since these taped regions are not included in the KT impedance eduction method, compliance of the tape is not expected to have an effect on the KT results.

For the reactance component, the error is much less than that observed for the conventional liner, except for frequencies near resonance. Since the reactance approaches zero in this frequency range, this is to be expected.

It is interesting to note the results at $800 \mathrm{~Hz}$ and Mach 0.0. Regardless of the liner length, the amount of attenuation is at least $10 \mathrm{~dB}$. Nevertheless, significant error is observed (Fig. 7) as the liner length is decreased. At Mach 0.3, the results for 600 and $800 \mathrm{~Hz}$ provide additional confirmation. The attenuation is larger at $600 \mathrm{~Hz}$, yet the error is also larger at this frequency, and is observed to increase as the liner length is decreased. This offers additional confirmation that the liner length is important to the CHE eduction process. The interactive effects of attenuation and liner length are explored further in the next section with the use of a conventional liner.

\section{B. Conventional liner results}

Similar data were acquired with the conventional liner mounted in the GFIT. Figure 9 compares impedance spectra educed with the CHE and KT methods with this liner in the absence of mean flow. The first spectrum (black squares) is educed with the CHE method for the full liner (no tape). The remaining spectra (red diamonds, green triangles, and blue circles) represent impedances educed with the CHE method when 2,4 , or 6 inches of tape are used to cover the trailing edge of the liner, respectively. The black ' $\mathrm{x}$ ' symbols represent impedances educed with the KT method for the full liner.

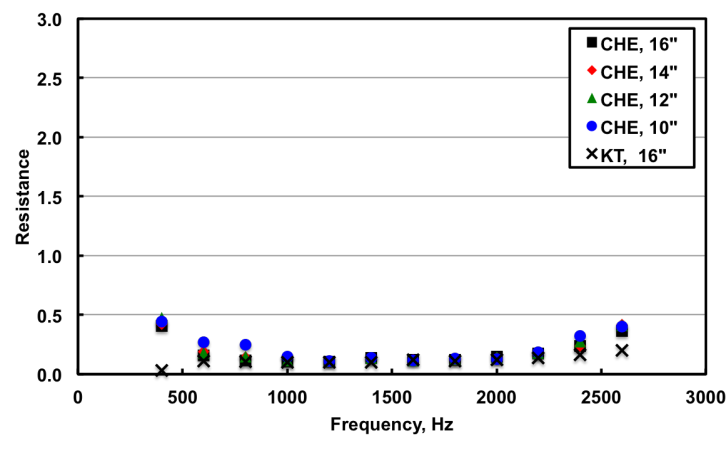

(a) Normalized resistance.

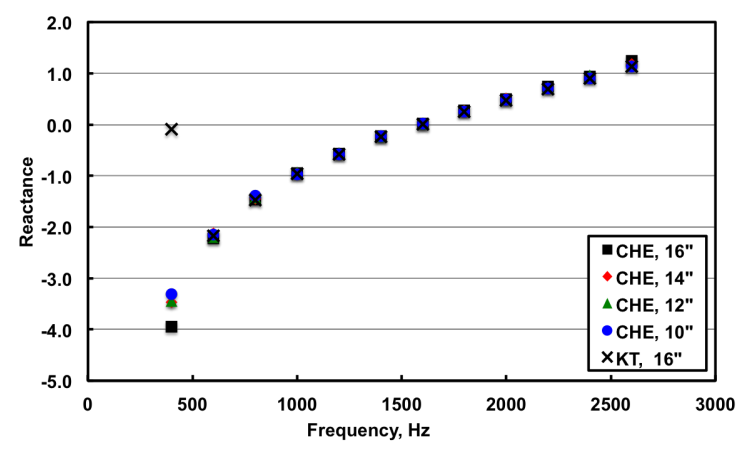

(b) Normalized reactance.

Figure 9: Comparison of impedance spectra educed with CHE and KT methods for liner lengths of 12 to 16 inches. Conventional liner; Mach 0.0.

A few items are evident in Fig. 9. First, the resistances educed with both methods for the full-length liner (16") compare favorably over the frequency range of 600 to $2200 \mathrm{~Hz}$. At $400 \mathrm{~Hz}$, the KT resistance has a marked decrease, whereas the CHE resistance has a significant increase. At the upper end of the frequency range, the CHE resistance experiences a larger increase than is observed with the KT method. An increase in resistance is expected at these frequencies, which are approaching the frequencies of anti-resonances. Nevertheless, although the difference between the methods is not large, further investigation is warranted. As the liner length is decreased via addition of tape, the resistance spectra educed with the CHE method gradually diverge from the full-liner result.

The reactance spectra compare favorably for frequencies of 600 to $2600 \mathrm{~Hz}$, for both impedance eduction methods and for all four liner lengths. At $400 \mathrm{~Hz}$, however, there is significant divergence of the results. The CHE reactances range from -4.0 to -3.3 , while the KT reactance is -0.1 . Based on the shape of the the spectrum above $400 \mathrm{~Hz}$, it seems clear that the magnitude of the reactance is approaching a maximum, 
indicating this frequency $(400 \mathrm{~Hz})$ is approaching an anti-resonance. Since the dominant component of reactance is given by $-\cot (k d)$, the reactance should transition from a large positive magnitude to a large negative magnitude at anti-resonance. This is one possible cause for the significant difference between the $\mathrm{KT}$ and $\mathrm{CHE}$ results at $400 \mathrm{~Hz}$, as the frequency regime near anti-resonance is known to be quite sensitive for impedance eduction methods.

Corresponding results for the Mach 0.3 condition are provided in Fig. 10. As should be expected for a conventional liner, the resistance increases noticeably. Similar to the results observed for the Mach 0.0 condition, the normalized resistance educed with the KT method is nearly zero at $400 \mathrm{~Hz}$. For this test condition, the separation between CHE and KT results is much more pronounced as the liner length is decreased. This separation is particularly significant at the lower frequencies (below $1000 \mathrm{~Hz}$ ), and is also quite sizable at the highest frequency $(2400 \mathrm{~Hz}$ for the flow condition; the first higher-order mode is cut on at $2600 \mathrm{~Hz}$ ). The Mach 0.3 reactance spectrum has a lower slope (flatter curve) than that observed at Mach 0.0. There is also much more difference between the KT and CHE results at the lower frequencies.

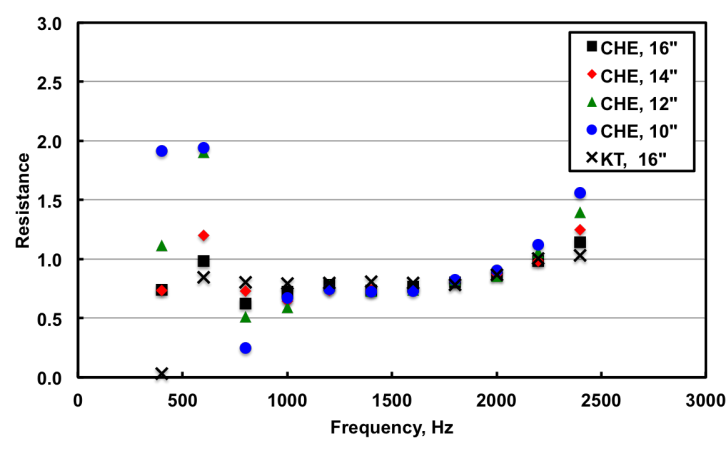

(a) Normalized resistance.

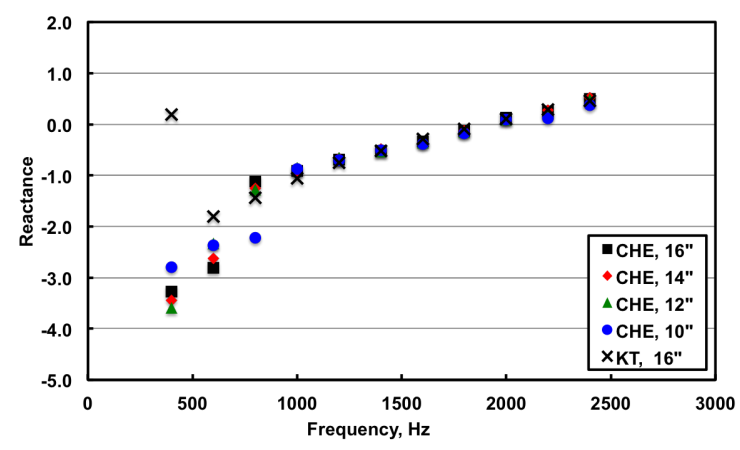

(b) Normalized reactance.

Figure 10: Comparison of impedance spectra educed with CHE and KT methods for liner lengths of 12 to 16 inches. Conventional liner; Mach 0.3.

Figure 11 provides a comparison of attenuation levels measured with the conventional liner installed in the GFIT. The attenuation at 1200 and $1400 \mathrm{~Hz}$ is greater for the Mach 0.0 condition than the Mach 0.3 condition. At other frequencies the Mach 0.3 condition provides the largest attenuations. Observe that the attenuation is quite small (approximately $5 \mathrm{~dB}$ or less) for the Mach 0.0 condition at frequencies at or below $1000 \mathrm{~Hz}$ and at or above $2000 \mathrm{~Hz}$. These frequencies correspond to those for which differences between the $\mathrm{KT}$ and CHE results are largest. Similar effects of limited attenuation are also observed in the Mach 0.3 data.

Figures 12 and 13 provide comparisons of the impedances educed with the CHE method for various liner lengths with the impedance educed with the KT method for the full (16") liner. As mentioned earlier, due to the difficulties experienced with the KT method at $400 \mathrm{~Hz}$, this comparison is limited to frequencies above $400 \mathrm{~Hz}$. The Mach 0.0 results presented in Fig. 12 clearly suggest an effect of liner length on the impedance eduction process. The error in resistance (Fig. 12a) increases with decreasing frequency below cut-off, and in most cases is inversely proportional to the liner length. It should be noted, however, that the error also increases (relative to the mid-frequency results) at the upper end of the frequency range. As was observed for the lower frequencies, it is apparent that the liner length affects the attenuation. The cause of this behavior at frequencies well above the cross-over frequency is not known. 


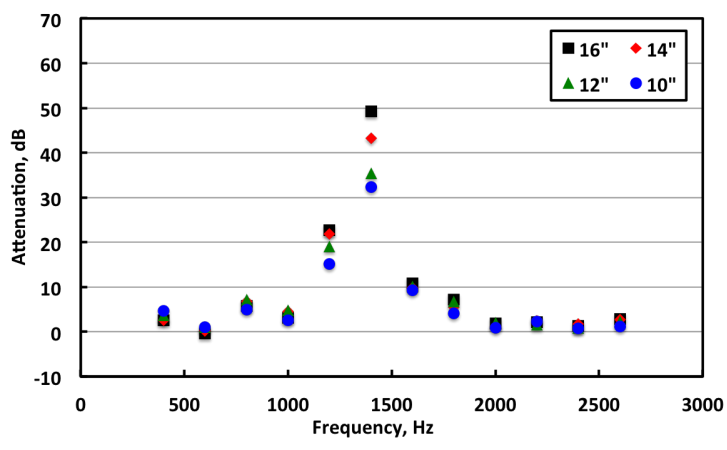

(a) Mach 0.0

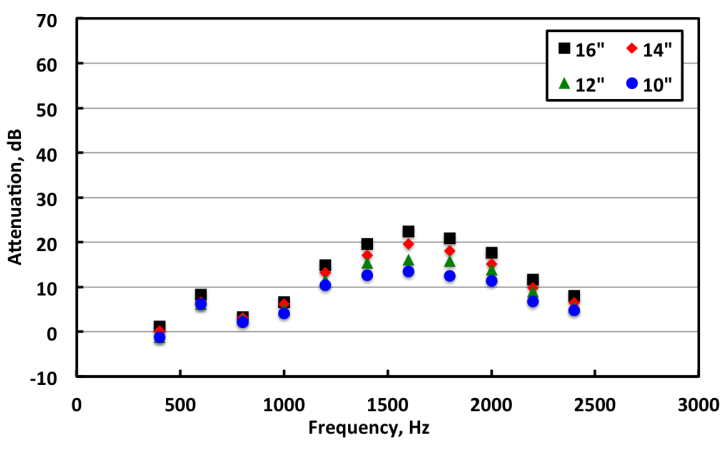

(b) Mach 0.3

Figure 11: Attenuation $[\operatorname{SPL}(0)-\operatorname{SPL}(L)]$ measured with conventional liner lengths of 10 to 16 inches.

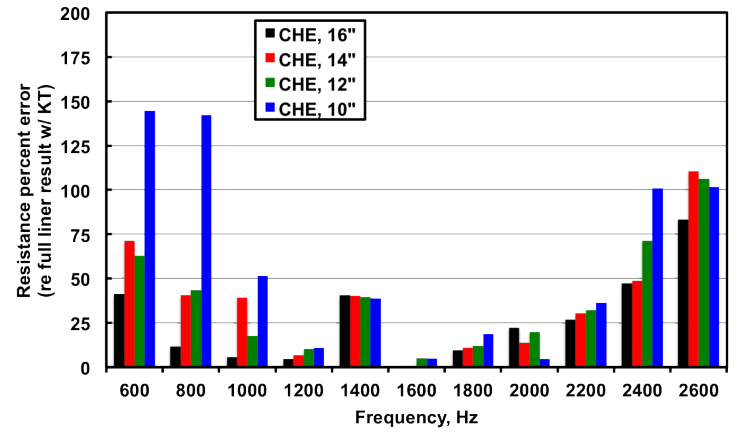

(a) Normalized resistance.

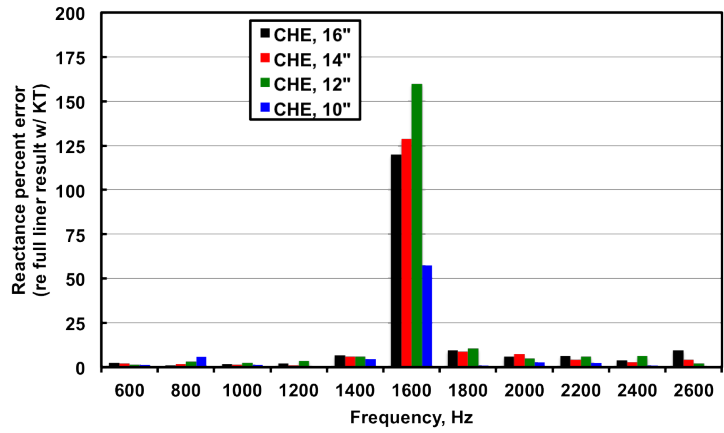

(b) Normalized reactance.

Figure 12: Comparison of CHE (for various liner lengths) and KT educed impedance spectra for full (16") conventional liner. Mach 0.0.

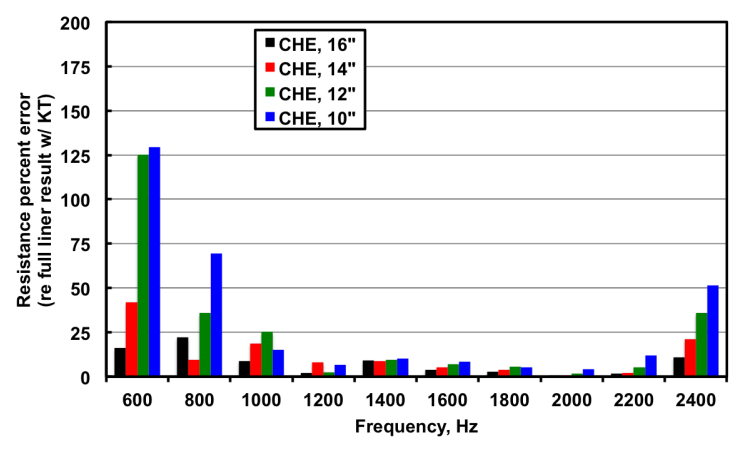

(a) Normalized resistance.

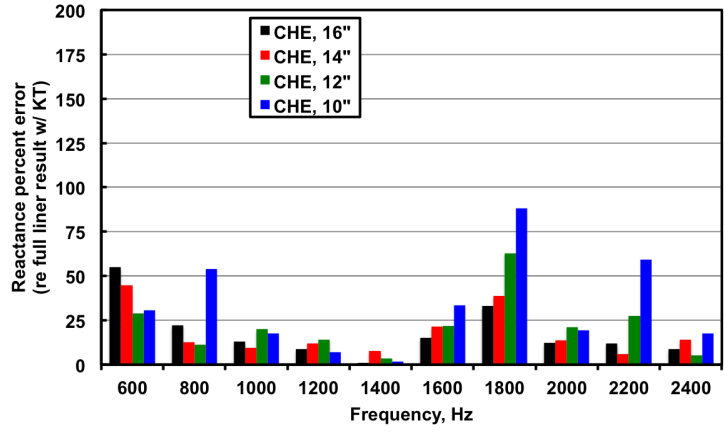

(b) Normalized reactance.

Figure 13: Comparison of CHE (for various liner lengths) and KT educed impedance spectra for full (16") conventional liner. Mach 0.3. 
One possible explanation for this general increase in percentage error relative to the wire mesh liner, especially at Mach 0.0 , is the fact that the measured resistance is much lower for the conventional liner than for the wire mesh liner. Hence, slight deviations in educed resistance represent much larger percentage changes than are experienced for the wire mesh liner. The importance of this observation should not be ignored. For the majority of liners considered for use in commercial aircraft engine nacelles, the desired normalized resistance is at least 0.5 (and often much higher) at typical mean flow velocities (nominally Mach 0.3 to 0.6 ). Therefore, the current results suggest that the CHE impedance eduction method is quite robust for typical applications. Regardless, it is helpful to better understand the limitations of this method.

In contrast, the reactance percent errors (Fig. 12b) do not correlate well with the liner length for the entire frequency range considered. The largest error occurs at $1600 \mathrm{~Hz}$, which corresponds to the resonance of the conventional liner. This sensitivity near resonance is to be expected, as the magnitude of the reactance is zero at resonance, thereby causing very small errors to be greatly magnified on a percentage scale.

Similar effects are observed for the resistances educed at the Mach 0.3 condition (Fig. 13a), although the errors are not quite as large over the entire frequency range. This lends credence to the supposition that, for the Mach 0.0 data, much of the error shown in Fig. 12a is due to the small values of resistance. At Mach 0.3, the error again grows at the lower frequencies, but is significantly subdued (relative to the Mach 0.0 results) at the higher frequencies. The corresponding reactance results (Fig. 13b) show an increase in error over the entire spectrum, with no strong correlation between liner length and error. The largest errors occur at 1800 and $2200 \mathrm{~Hz}$, which are close to the resonance of the liner.

The Mach 0.3 results at 600 and $800 \mathrm{~Hz}$ provide interesting insights into the effects of attenuation and liner length. Note that the attenuation at $600 \mathrm{~Hz}$ is at least twice that observed at $800 \mathrm{~Hz}$, whereas the error is markedly higher at $600 \mathrm{~Hz}$. This suggests that, although a significant portion of impedance eduction error is due to reductions in attenuation, there is clearly another contributor to the error. These results suggest that liner length is at least a secondary contributor to impedance eduction error with the CHE method.

\section{Concluding Remarks}

This study explored the effects of liner length and attenuation on the CHE impedance eduction method. Two acoustic liners, a wire mesh-over-honeycomb liner and a conventional, perforate-over-honeycomb liner, were used for this study. Each liner was tested in the NASA Langley Grazing Flow Impedance Tube (GFIT), and the resultant data were processed using two impedance eduction methods. The first is the CHE method, an indirect impedance eduction method for which the surface impedance of an acoustic liner is inferred through an iterative process based on repeated solutions to the convected Helmholtz equation. The second is the KT method, a direct method that uses the Kumaresan and Tufts algorithm to compute the impedance directly. The KT method only employs data measured over the length of the liner and well away from the leading and trailing edges of the liner, thereby minimizing the effects of reflections. Results achieved with the KT method for each full-length liner were used as a baseline against which impedances educed with the CHE method were compared as the liner was repeatedly shortened by the addition of aluminum tape at the trailing edge. Clearly, the value of this comparison is dependent upon the validity of the impedances educed with the KT method. A comparison of results achieved with the two impedance eduction methods is used to explore the interactive effects of liner length and attenuation on the CHE impedance eduction method. Some of the key results of this study are:

1. For the majority of test conditions, the impedances educed with the two methods compare favorably.

2. The KT method provides anomalous results at $400 \mathrm{~Hz}$, so results educed at this frequency are not used for comparisons of the two impedance eduction methods. This anomalous behavour is expected to be the subject of a future investigation.

3. For those test conditions where the attenuation is low (nominally less than $10 \mathrm{~dB}$ ) over the length of the liner, there is a resultant increase in the difference between the impedances educed with the CHE and KT. This difference is typically larger for the resistance than for the reactance.

4. As should be expected, the 'percent error' (defined as the difference between the CHE and KT results divided by the KT result) increases when the absolute value of the measured quantity (resistance or reactance) becomes very low. For conventional liners, this typically occurs with the resistance component 
for the no-flow (Mach 0.0) test condition, whereas the resistance increases and the percent error decreases for the Mach 0.3 test condition. For frequencies near resonance, the reactance approaches zero, and the CHE method correspondingly experiences an increase in percent error although the absolute error is rather small.

5. For the majority of the results discussed herein, there is a definite effect of liner length on the CHE impedance eduction method. This effect is less important than that due to limited attenuation, but cannot be ignored. For frequencies sufficiently low that the wavelength is longer than the liner length, the effects of liner length are clearly evident. For the most part, the percent error increases as the liner length decreases.

6. There is a strong correlation between decreased liner length and increased error in the CHE impedance eduction method across the entire frequency range. Since this effect is not confined to the lower frequencies for which the wavelength is larger than the liner length, this implies that at least a portion of this error is directly related to the number of wavelengths that occur within the axial extent of the liner. This effect is further magnified when the liner provides poor attenuation.

7. Based on the very limited results provided in this study, the minimum acceptable liner length for use in the GFIT is approximately 16 inches, but the optimum length is 24 inches, the maximum possible.

\section{Acknowledgments}

The authors wish to express sincere appreciation to Carol Harrison, Martha Brown, and Brian Howerton for their efforts in the acquisition of the GFIT data. The Advanced Air Transportation Technologies Project of NASA's Advanced Air Vehicle Program funded this work.

\section{References}

\footnotetext{
${ }^{1}$ Armstrong, D. L., Beckemeyer, R. J., and Olsen, R. F., "Impedance Measurements of Acoustic Duct Liners With Grazing Flow," Paper presented at 87th Meeting of the Acoustical Society of America 1999-1864, 1974.

${ }^{2}$ Watson, W. R., Jones, M. G., and Parrott, T. L., "Validation of an Impedance Eduction Method in Flow," AIAA Journal, Vol. 37, No. 7, July 1999, pp. 818-824.

${ }^{3}$ Jones, M. G., Watson, W. R., Tracy, M. B., and Parrott, T. L., "Comparison of Two Waveguide Methods for Educing Liner Impedances in Grazing Flow," AIAA Journal, Vol. 42, No. 2, Februrary 2004, pp. 232-240.

${ }^{4}$ Watson, W. R., Tanner, S. E., and Parrott, T. L., "Optimization Method for Educing Variable-Impedance Liner Properties," AIAA Journal, Vol. 36, No. 1, 1998, pp. 18-23.

${ }^{5}$ Watson, W. R., Gerhold, C. H., Jones, M. G., and June, J. C., "Single Mode Theory for Impedance Eduction in LargeScale Ducts with Grazing Flow," AIAA Paper 2014-3351, June 2014.

${ }^{6}$ Watson, W. R. and Jones, M. G., "A Comparative Study of Four Impedance Eduction Methodologies Using Several Test Liners," AIAA Paper 2013-2274, May 2013.

${ }^{7}$ Watson, W. R., Carpenter, M. H., and Jones, M. G., "Performance of Kumaresan and Tufts Algorithm in Liner Impedance Eduction with Flow," AIAA Journal, Vol. 53, No. 4, April 2015, pp. 1091-1102.

${ }^{8}$ Jones, M. G., Watson, W. R., Tracy, M. B., and Parrott, T. L., "Comparison of Two Acoustic Waveguide Methods for Determining Liner Impedance," AIAA Paper 2001-2266, May 2001.

${ }^{9}$ Jones, M. G., Watson, W. R., and Parrott, T. L., "Benchmark Data for Evaluation of Aeroacoustic Propagation Codes with Grazing Flow," AIAA Paper 2005-2853, May 2005.

${ }^{10}$ Watson, W. R., Jones, M. G., and Parrott, T. L., "Investigation of an Anomaly Observed in Impedance Eduction Techniques," AIAA Paper 2008-3013, May 2008.

${ }^{11}$ Watson, W. R. and Jones, M. G., "Explanation of Anomalous Behavior Observed in Impedance Eduction Techniques Using Measured Data," AIAA Paper 2010-3766, May 2010.

12 Jones, M. G. and Watson, W. R., "Validation of an Improved Experimental Method for Use in Impedance Eduction," AIAA Journal, Vol. 51, No. 1, January 2013, pp. 186-199.

${ }^{13}$ Jones, M. G., Watson, W. R., and June, J. C., "Optimization of Microphone Locations for Acoustic Liner Impedance Eduction," AIAA Paper 2015-3271, June 2015.

${ }^{14}$ Bendat, J. S. and Piersol, A. G., Random Data: Analysis and Measurement Procedures, Wiley-Interscience, 1971.

${ }^{15}$ Myers, M. K., "On the Acoustic Boundary Condition in the Presence of Flow," Journal of Sound and Vibration, Vol. 71 , No. 3, 1980, pp. 429-434.

${ }^{16}$ G. W. Stewart, I., "A Modification of Davidon's Minimization Method to Accept Difference Approximations of Derivatives," Journal of ACM, Vol. 14, No. 1, 1967, pp. 72-83.
} 